\title{
Malignant Colorectal Polyps; Pathological Consideration (A review)
}

\author{
Bita Geramizadeh ${ }^{1}$, Mahsa Marzban ${ }^{2}$, David A. Owen ${ }^{3}$ \\ 1. Dept. of Pathology, Transplant Research Center, Shiraz, Iran \\ 2. University of British Columbia, Vancouver, BC, Canada \\ 3. Dept. of Pathology, Vancouver General Hospital and University of British Columbia, Vancouver, BC, Canada
}

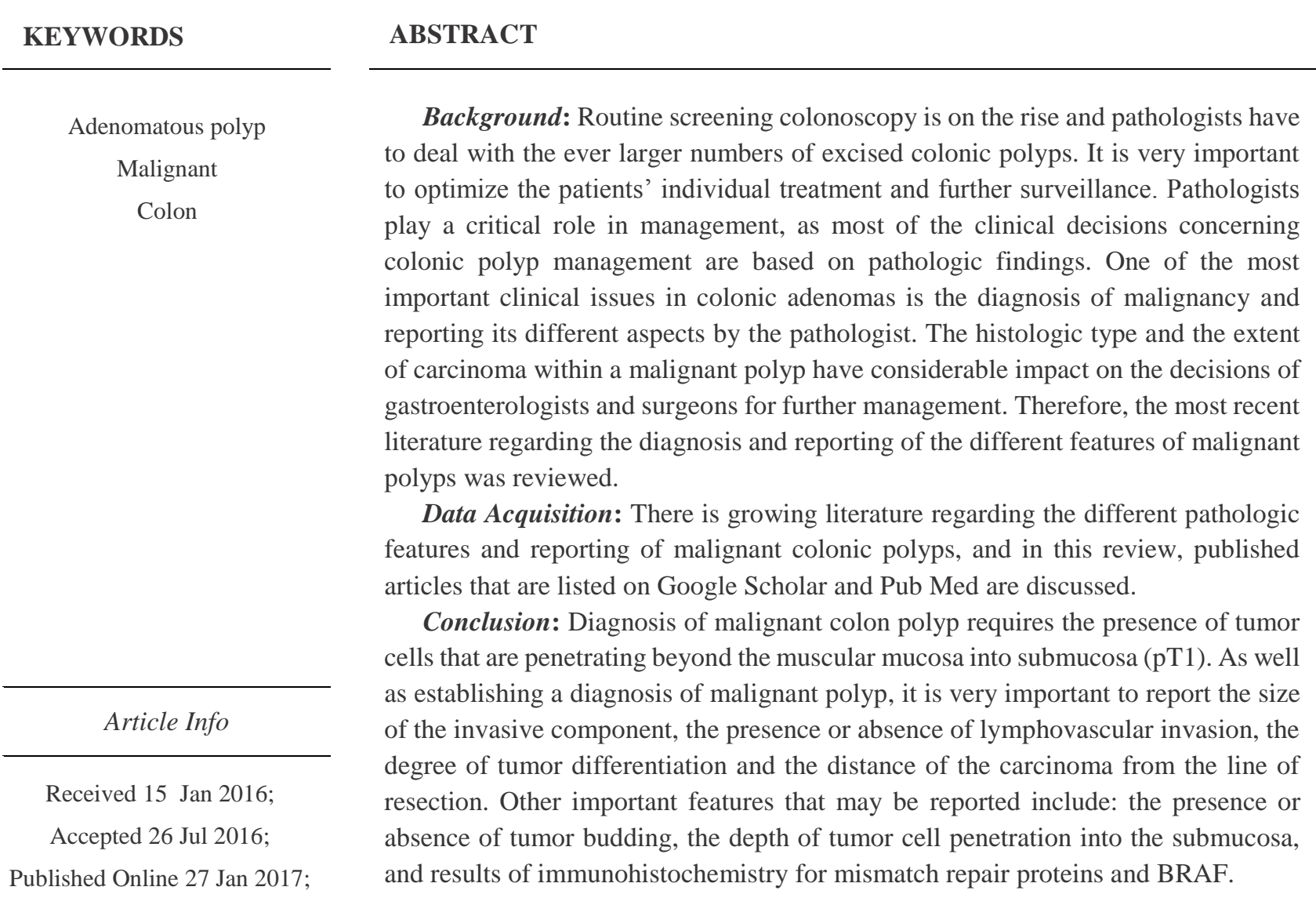

Corresponding Information: Dr. Bita Geramizadeh, Dept of Pathology, Transplant Research Center, Shiraz University of Medical Sciences, Shiraz, Iran. Tel: +98987136473238 Email: geramib@sums.ac.ir

Copyright ( 2017, IRANIAN JOURNAL OF PATHOLOGY. This is an open-access article distributed under the terms of the Creative Commons Attribution-noncommercial 4.0 International License which permits copy and redistribute the material just in noncommercial usages, provided the original work is properly cited.

\section{Introduction}

Colorectal polyps are common in the general population and most polyps are adenomas. In screening colonoscopic studies, the reported prevalence of adenomas was $24 \%$ to $48 \%$. The main importance of adenomas is their relationship with colorectal cancer, as most cancers arise from these precursor lesions (1). Over the past decades, the number of screening colonoscopies is increasing and more adenomas are being identified. These need to be removed and this can usually be done endoscopically. However, some of these adenomas are large, complicated, or malignant, and safe removal may require advanced procedures, such as surgical resection (2).

Pathologic findings play an important and critical role in clinical decision-making for the proper management of malignant colorectal polyps (3).

Some malignant polyps can be treated successfully by endoscopic resection alone; however, this decision depends completely on histopathological criteria that can predict tumor 
aggressiveness and the risk of recurrence or lymph node and distant metastasis (4).

Accordingly, it is important for all polyps to be submitted for histopathological examination, and the interpretation of malignant colon polyps by pathologists should be correct and complete (5). In this review the criteria for the diagnosis of malignant colorectal polyps was described and histologic features that must be included in the pathology report were emphasized, i.e. findings that influence management, and determine the risk of recurrence or distant metastasis.

\section{Definition}

Colorectal malignant polyps (pT1 tumor), according to Tumor-Nodes-Metastases (TNM) classification are cancerous polyps that have invaded the sub-mucosal layer. Therefore, a malignant large bowel polyp is an adenoma, in which an invasive carcinoma develops and spreads to involve the submucosa of the polyp's head or stalk or in the case of a sessile polyp, the submucosa of the underlying bowel wall (5). While adenomatous polyps can harbor high-grade dysplasia and other non-invasive histologic features, invasion through the muscularis mucosa but limited to the submucosa (pT1) is by definition indicative of an adenocarcinoma (malignant polyp) (6).

\section{Incidence}

According to some recent publications, malignant polyps account for up to $12 \%$ of endoscopically removed polyps. Accurate diagnosis is therefore a critical point in the prevention of colorectal cancer and permits use of advanced polyp removal techniques, such as Endoscopic Mucosal Resection (EMR), Endoscopic Submucosal Dissection (ESD), or even open surgery (7).

\section{Diagnosis}

With the above-mentioned methods of polypectomy, there is a good opportunity to excise the polyp completely instead of in a piecemeal fashion (8). This also facilitates a more accurate histological examination. The polyp may have had a benign appearance at endoscopy, yet after pathology examination, it may be found to have an invasive focus of adenocarcinoma. Furthermore, patient evaluation is more complicated if the polypectomy site has not been marked (usually by an India ink tattoo) during the initial endoscopy. In this situation, risk assessment of the possibility of residual or recurrent disease and lymph-node metastasis requires balancing the risk of recurrent disease against the patient's operative risk for further surgery. This assessment may be difficult and requires a multidisciplinary approach (9).

Based on the pathologist's report, the clinician would make a decision on whether polypectomy alone is an adequate therapy or whether the patient needs to undergo a subsequent definitive surgical resection (2).

Therefore, after the primary polypectomy, the pathologists' responsibility is to diagnose the case accurately, while producing a complete and informative report that enables an assessment of the risks for the presence or development of residual and metastatic disease (10).

\section{Gross Handling of the resected polyps}

The polypectomy specimen should be placed in adequate formalin in the endoscopy room (typically ten times the volume of formalin to the volume of the specimen). The polyps need a minimum of 2 to 3 hours of fixation; however, this depends on the size of the polyp. Generally, polyps larger than 1.5 $\mathrm{cm}$ need overnight fixation (2).

Adequately-fixed polyps are not friable and feel firm on palpation (11).

After inspection and description recording including the exact size, the next step is identifying the margin of resection. This needs to be inked. The margin will be the most distal end of the stalk in pedunculated polyps or the line of resection (base of the polyp) in sessile polyps (12). At some centers, after polypectomy, the endoscopists themselves may identify the line of resection by pin or application of India ink (2). The next step is 
dissection of the polyp. The preferred method for polyps larger than $0.9 \mathrm{~cm}$ is dissection through a sagittal section perpendicular to the stalk or line of resection, to preserve the anatomical landmarks (13). However, according to the authors' personal experience, for polyps less than 0.4 , it is better not to section them and embed the whole polyp in one cassette. For polyps between 0.4 and $0.9 \mathrm{~cm}$, they can be bisected with both sections placed in one cassette. For polyps between $0.9 \mathrm{~cm}$ and $1.2 \mathrm{~cm}$, the best method is to trisect the specimen by shaving the two sides and then placing the central part in one cassette and the two shaved sides in an additional cassette. For polyps larger than $1.2 \mathrm{~cm}$, it is best to cut as many sections as appropriate to submit the central stalk area in a separate cassette (14).

Another approach, which can only be used in polyps with a stalk, is taking a transverse section from the most distal part of the stalk and then dissection of the polyp. Whatever the dissection method, the entire specimen should be embedded (15).

Fragmentation of the polyp should be mentioned in the gross description, because the evaluation of the actual size, invasion and involvement of the line of resection may not be accurate in a fragmented polypectomy specimen $(11,12,13)$.

\section{Pathologic Diagnosis and Reporting}

Diagnosis of a malignant colon polyp: High grade dysplasia is defined as the presence of nuclear stratification and/or cytologic features of carcinoma such as irregular branching, budding, and cribriform appearance of the crypts, loss of cytoplasmic mucin, nuclear hyperchromasia and vesicular nuclei with nucleoli. There is no associated desmoplastic stromal response (15). For the diagnosis of a malignant polyp, by definition, the main step is identifying tumor cells with the above-mentioned characteristics below the muscularis mucosae and extending into the submucosa. This type of adenoma should be reported as a malignant polyp (pT1) (2).

According to the World Health Organization (WHO) recommendation, the term high-grade dysplasia should be used instead of "intramucosal carcinoma" for adenomas in which there is mucosal invasion (i.e. invasion of lamina propria \pm muscularis mucosae), yet with no extension below the muscularis mucosae. The reason for this designation is that mucosal invasion alone (i.e. without submucosal invasion) is associated with a negligible risk of malignancy (lymph node spread). This is a consequence of the lymphatic drainage of the colon where, no lymphatic channels are located superficial to the muscularis mucosae. Therefore, focal cancer that has not invaded through the muscularis mucosae presents no risk of lymph node spread. Normally, lymphatic channels are found in the superficial submucosa and within the muscularis mucosa, yet with only rare extensions into the lamina propria (mucosa) and are limited to the base of the crypts. This near-absence of lymphatics within the mucosa is the reason for the observed lack of malignant potential (lymph node metastasis) in polyps showing only high-grade dysplasia (formerly called intramucosal carcinoma). Therefore, the majority of these lesions do not require further surgery (16). In cases where there is mucosal invasion, besides the use of the term "high-grade dysplasia" it is recommended to insert a comment to explain the findings and their significance $(16,17)$.

Additional findings in the report: There are some other important findings, which have a great impact on the patient's outcome, management, and surveillance and should be included in the pathology report of a malignant polyp. These findings have been shown to have a good correlation with the risk of lymph node involvement, recurrence and metastasis:

1) Presence or absence of any amount of poorlydifferentiated adenocarcinoma.

Three tumor grades have been described for malignant colorectal tumors. Grade 1 corresponds to a well-differentiated intestinal-type adenocarcinoma and is composed of well-formed glands with open lumina or with more than $95 \%$ glandular differentiation. Grade 2 is moderately differentiated intestinal-type adenocarcinoma containing solid nests showing only focal glands or $50 \%$ to $95 \%$ glandular differentiation. In Grade 3, the carcinoma is a poorly differentiated intestinal-type, signet ring cell or mucinous adenocarcinoma, composed of hyperchromatic cells arranged into solid sheets and 
forming absorptive glands. These tumors have less than $50 \%$ glandular differentiation (16).

2) Presence or absence of angiolymphatic invasion: The presence of tumor cells within a true endothelial-lined channel in the absence of red blood cells is defined as lymph vessel invasion. The occurrence of lymph vessel invasion is generally found in polyps with a positive surgical margin and/or the presence of poorly differentiated tumor (18). It is not recommended to perform immunohistochemistry for the routine confirmation of the lymphovascular invasion; however specific marker for lymph vessel endothelial cells, i.e. D240 , can be helpful in equivocal cases (2).

Another issue to be considered is venous invasion, which is less well studied than lymphatic invasion, and may have an impact on the prognosis and lymph node involvement. It is recommended to record the presence or absence of venous invasion in the pathologic report. Sometimes endothelial immunohistochemical markers, such as CD31 and CD34, are helpful for confirming the presence of tumor cells within an endothelial-lined vessel (2).

According to the authors' experience, angiolymphatic invasion is very difficult to assess, because the vessels are hard to differentiate from retraction artefact, yet as mentioned, this most commonly accompanies poorly differentiated tumors with positive margins.

3) Distance of invasive adenocarcinoma from margin of resection (a distance of $1 \mathrm{~mm}$ or less is considered to represent a positive margin). This finding is closely correlated with the risk of recurrence (16). Therefore, it is recommended to report the distance of tumor cells from the resection margins, which is also the cauterized margin (2) (Figure 1).

4) Another feature, which is currently recommended to be recorded, in the pathologic report is high-grade tumor budding, which is now considered as an important predictor of lymph node metastasis in malignant polyps. Tumor budding is defined as "presence of de-differentiated single cells or small clusters of up to 5 cells at the invasive front of colorectal cancer or malignant polyp". Highgrade tumor budding is considered to be present when there are more than 10 tumor buds in any microscopic field viewed in a $25 \mathrm{X}$ field $\left(0.385 \mathrm{~mm}^{2}\right)$ $(19,20,21,22)$ (Figure 2).

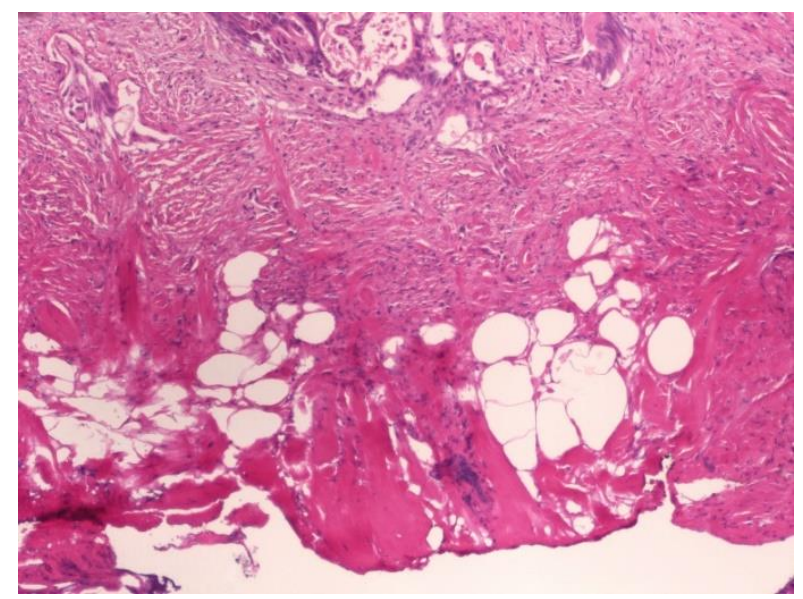

Fig 1. Base of a Malignant Polyp.

There is heavy cautery artefact but it is still possible to measure the distance between the margin of excision and the edge of the carcinoma.

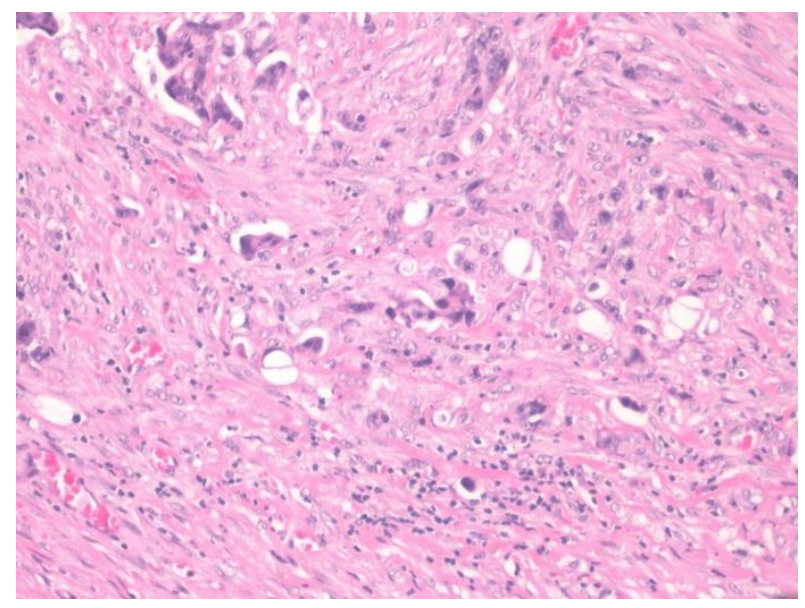

Fig 2. High-Grade Tumor Budding

Numerous clumps of tumor cells are present, each of which contains less than five cells. There are more than ten tumor buds in this $25 \mathrm{X}$ field.

5) There is no recommendation to report the level of invasion in malignant polyps; however some authors continue to report this, because earlier studies showed a good correlation between the level of invasion and lymph node involvement. Haggitt et al. classified the level of invasion in a pedunculated malignant polyp as level 1 to 4 . Level 1 describes invasive adenocarcinoma limited to the polyp head; level 2 includes neck involvement; level 3 corresponds to adenocarcinoma cells in the stalk; and level 4 to adenocarcinoma cells infiltrating the submucosa at the level of the adjacent bowel wall 
(23). This classification is difficult for application in sessile polyps, so other classifications have been proposed by some authors. The degree of submucosal invasion in sessile polyps has been classified to three types based on the depth of invasion. When less than one-third of the submucosa is invaded, the stage is sm1, and if more than two-thirds is invaded the stage is sm3, while stage sm2 is intermediate with invasion of cancer into the middle third $(24,25,26)$. However, so far, there is no consensus to report these levels and currently, they do not have any impact on the management and surveillance of the patients with malignant colonic polyps (16).

Molecular biomarkers in malignant colon polyps: Colorectal tumors are a heterogeneous group of diseases that arise and evolve through the stepwise accumulation of differing sets of genetic and epigenetic alterations (27). Recent advances in molecular biomarkers to personalize therapy contributed to major progress in the treatment and prognosis of the disease, and many of them are now part of routine laboratory tests (28). There are only a few studies about adding immunohistochemical biomarkers of colon cancers in malignant polyps, however, according to some reports, about $46.8 \%$ exhibited KRAS mutation, 6.5\% BRAF mutations and $10.6 \%$ were MSI-H. Many of the gastrointestinal pathology centers dealing with colorectal polyps are now routinely performing these tests by immunohistochemical methods (Testing for MSI-H by immunostains for MLH-1, MSH-2, PMS-6 and MSH-6). (29, 30, 31)

Pitfall in the diagnosis of invasive component (Pseudo-invasion):

The most important pitfall, which causes incorrect and over diagnosis of malignant colon polyps is the presence of misplaced glands below the muscularis mucosae (Figure 3). Most polyps with pseudoinvasion are large and pedunculated lesions, which are typically located in the sigmoid colon. They can rarely be seen in the rectum $(32,33)$. They represent prolapse of the adenomatous epithelium into the polyp stalk $(33,34)$. It is believed that they are caused by herniation of the intramucosal tissue into the submucosa when the polyp stalk is twisted, thus, they are frequently accompanied by evidence of previous bleeding (hemosiderosis) $(34,35)$. If the submucosal tumor nodule seen in a polypoid tumor is purely a result of herniation, it should be discriminated from true invasion because nonmalignant polyps with pseudoinvasion never cause distant metastasis (34).

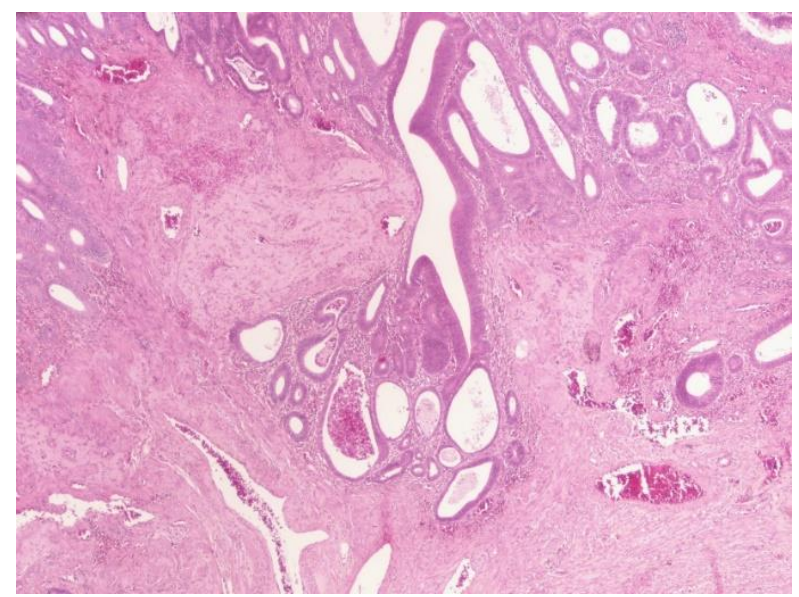

Fig 3. In this polyp misplaced epithelium is present in the submucosa immediately adjacent to a large submucosal vessel (pseudoinvasion). However, note that the epithelium is accompanied by normal appearing lamina propria.

The submucosal glands are rounded with no infiltrative border and they are always surrounded by lamina propria and do not have cytoarchitectural features of malignancy. There is hemorrhage and hemosiderin in the surrounding submucosa and no desmoplastic stroma in misplaced glands (pseudoinvasion). Admixed dilated normal glands may also be present in the submucosa and there may be extravasation of acellular mucin pools with regular borders (Figure 4). Ischemic changes (granulation tissue, erosions, and exudates) are often present at the surface of the polyp due to polyp torsion. Invasive adenocarcinoma is usually associated with high grade dysplasia and desmoplasia. According to the authors' personal experience, this stromal desmoplasia is a very helpful distinguishing feature. Pools of mucin may be present; however these may show irregular borders and often contain isolated dysplastic cells within the mucin pool $(33,34,35,36,37)$. 


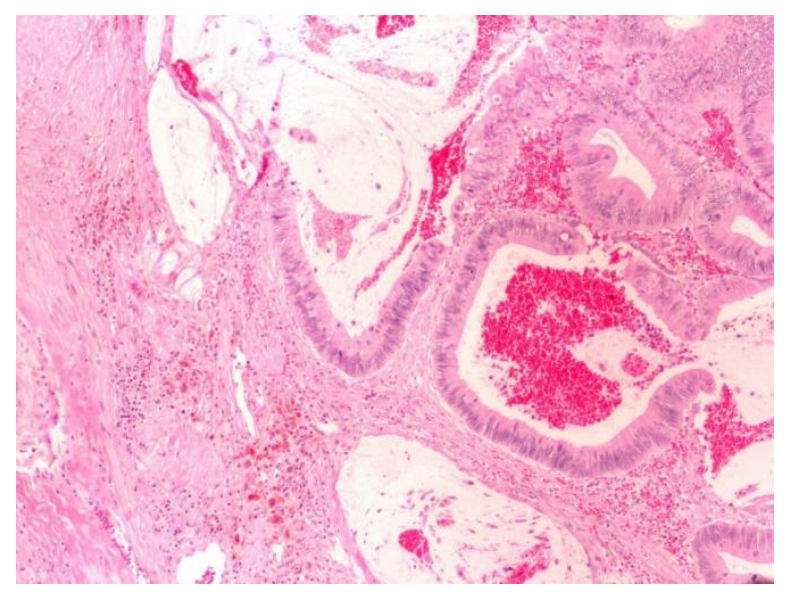

Fig 4. Probably because of polyp torsion, the misplaced epithelium has accumulated inspissated mucin and the crypts have ruptured releasing pools of acellular mucin into the polyp stalk.

In some studies, immunohistochemistry has been used for differential diagnosis of misplaced glands versus true invasion. Yantiss et al. have reported increased staining of the submucosal epithelium for Matrix Metalloproteinase 1 (MMP-1) and/or p53, combined with decreased staining of the submucosal epithelium for membranous E-cadherin, for establishing a diagnosis of adenocarcinoma (38).

\section{Conclusion}

Pathologists play a critical role in the diagnosis and management of malignant colon polyps, thus pathologic reports should be as informative and complete as possible and contain all the information the clinician needs for further management and surveillance.

\section{Conflict of Interests}

The authors declare that there is no Conflict of Interests.

\section{References}

1. Geramizadeh B, Keshtkar-Jahromi M. Pathology of Colorectal Polyps: A Study from South of Iran. Annals of Colorectal Research. 2013; 1: 60-2.

2. Cooper HS. Pathology of the endoscopically removed malignant colorectal polyp. Current Diagnostic Pathology 2007; 13, 423-437

3. Costantini M, Sciallero S, Giannini A, Gatteschi B, Rinaldi $P$, Lanzanova $G$, et al. Interobserver agreement in the histologic diagnosis of colorectal polyps: the experience of the multicenter adenoma colorectal study (SMAC). J Clin Epidemiol 2003;56: 209-214

4. Rex DK, Alikhan M, Cummings O, Ulbright TM. Accuracy of pathologic interpretation of colorectal polyps by general pathologists in community practice. Gastrointest Endosc 1999;50:468-74

5. Komuta K, Batts K, Jessurun J, Snover D, GarciaAguilar J, Rothenberger D, Madoff R. Interobserver variability in the pathological assessment of malignant colorectal polyps. Br J Surg 2004; 91: 1479-1484

6. Aarons CB, Shanmugan S, Bleier JI. Management of malignant colon polyps: Current status and controversies. World J Gastroenterol 2014; 20: 1617816183

7. Ueno H,Mochizuki H, Hashiguchi Y, Shimazaki H, Aida S, Hase K, Matsukuma S, et al. Risk Factors for an Adverse Outcome in Early Invasive Colorectal Carcinoma. Gastroenterology 2004;127:385-394

8. Rickert A, Aliyev R, Belle S, Post s, Kienle P, Kähler G,Oncologic colorectal resection after endoscopic treatment of malignant polyps: Does endoscopy have an adverse effect on oncologic and surgical outcomes? Gastrointest Endos 2014;79,951-60

9. Nienstedt R, De Carli N, Monica F, Guido F, Guido $\mathrm{F}$, Valiante $\mathrm{F}$, et al. The management of malignant polyps in colorectal cancer screening programmes: A retrospective Italian multi-centre study. Dig and Liver Dis 2015;47:715-719

10. Morson BC, Whiteway JE, Jones EA, Macrae FA, Williams CB. Alimentary tract and pancreas Histopathology and prognosis of malignant colorectal poiyps treated by endoscopic polypectomy.Gut 1984; 25 : 437-444

11. Cranley JP, Petras RE, Carey WD, Paradis K, Sivak MV..When is endoscopic polypectomy adequate 
therapy for colonic polyps containing invasive carcinoma? Gastroenterology. 1986 ;91(2):419-27

12. Coverlizza S, Risio M, Ferrari A, FenoglioPreiser M, Rossin FP. Colorectal Adenomas Containing invasive Carcinoma. Cancer 64: 1937-1947, 1989

13. Cooper HS, Deppisch LM, Gourley WK, Kahn EI, Lev R, Manley PN, Pascal RR, Qizilbash AH, Rickert RR, Silverman JF, et al. Endoscopically removed malignant colorectal polyps: clinicopathologic correlations. Gastroenterology. 1995;108:1657-65

14. Lester SC. Gastrointestinal, Hepatobiliary and pancreatic specimens. In: Manual of surgical pathology. $3^{\text {rd }}$ edition. Philadelphia: Saunders;2010.p.183

15. Euscher ED, Niemann TH, Lucas JG, Kurokawa AM, Frankel WL. Large Colorectal Adenomas. An Approach to Pathologic Evaluation. Am J Clin Pathol 2001;116:336-340

16. Fogt F, Zimmerman RL, Ross HM, Daly T, Gausas RE. Identification of lymphatic vessels in malignant, adenomatous and normal colonic mucosa using the novel immunostain D2-40.Oncol Rep 2004;11:47-50.

17. Bosman FT, Carneiro F, Hruban RH, Theise ND. WHO Classification of Tumours of the Digestive System. 4th Ed. IARC Press, Lyon 2010.

18. Kaiserling E, Kröber S, Geleff S. Lymphatic vessels in the colonic mucosa in ulcerative colitis. Lymphology 2003 ;36:52-61.

19. Koelzer VH, Zlobec I, Lugli A. Tumor budding in colorectal cancer-ready for diagnostic practice? Human Pathol 2015, article in press

20. Graham RP, Vierkant RA, Tillmans LS, Wang ZAH, Laird PW, Weisenberger DJ, et al. Tumor budding in colorectal carcinoma confirmation of prognostic significance and histologic cutoff in a population-based cohort. Am J Surg Pathol 2015;39:1340-1346

21. Caie P, Turnbull Ak, Farrington SM, Oniscu A, Harrison DJ. Quantification of tumour budding, lymphatic vessel density and invasion through image analysis in colorectal cancer.J Transl Med 2014, 12:156168

22. Landau MS, Hastings SM, Foxwell TJ, Luketich JD, Nason KS, Davison JM. Tumor budding is associated with an increased risk of lymph node metastasis and poor prognosis in superficial esophageal adenocarcinoma. Mod Pathol. 2014 ; 27: 1578-1589
23. Ramirez M, Schierling S, Papaconstantinou HT, Thomas SJ. Management of the, malignant Polyp. Clinics in Colon and rectal Surgery 2008;21: 286-90

24. Haggitt RC, Glotzbach RE, Soffer EE, Wruble LD. Prognostic factors in colorectal carcinomas arising in adenomas: implications for lesions removed by endoscopic polypectomy. Gastroenterol 1985;89:328-36.

25. Kudo S. Endoscopic mucosal resection of flat and depressed types of early colorectal cancer. Endoscopy 1993;25: 455-61

26. Kikuchi R, Takano M, Takagi K, Fujimoto N, Nozaki R, Fujiyoshi T, Uchida Y. Management of early invasive colorectal cancer. Risk of recurrence and clinical guidelines. Dis Colon Rectum 1995;38:1286-95.

27. Lochhead P, chan AT, Giovannucci E, Fuchs CS, Wu K, Nishihara R, et al. Progress and Opportunities in Molecular Pathological Epidemiology of Colorectal Premalignant Lesions. Am J Gastroenterol. 2014 ; 109: 1205-1214

28. Geramizadeh B. Molecular Biomarkers of Colorectal Cancer: A review of published articles from Iran. Ann Colorectal Res. 2015; 3: e30100.

29. Yamane LS, Scapulatempo-Neto C, Alvarenga L, Oliveira CZ, Berardinelle GN, Almodova E, et al. KRAS and BRAF mutations and MSI status in precursor lesions of colorectal cancer detected by colonoscopy. ONCOLOGY REPORTS 2014; 32: 1419-1426

30. Omidifar N, Geramizadeh B, Mirzai M. K-ras Mutation in Colorectal Cancer, A report from southern Iran. IJMS 2015;40:454-60

31. Javadi F, Geramizadeh B, Mirzai M. BRAF Gene Mutation Analysis in Colorectal Cancer in South of Iran. Annals of Colorectal Research. 2014 June; 2(2): e19917

32. Bujanda L, Cosme A, Gil I, Arenas-Mirave JI. Malignant colorectal polyps. World J Gastroenterol 2010; 16: 3103-3111

33. Byun TJ, Han DS, Ahn SB, Cho HS, Eun AS, Cheol Y, et al. Pseudoinvasion in an adenomatous polyp of the colon mimicking invasive colon cancer. Gut and Liver 2009;3: 130-133

34. Tanizawa T, Seki T, Nakano M, kamiyama R. Pseudoinvasion of the colorectal polypoid tumors: Serial section study of problematic cases. Pathology International 2003;53: 584-590 
35. Shepherd NA, Griggs RKL. Bowel cancer screening-generated diagnostic conundrum of the century: pseudoinvasion in sigmoid colonic polyps. Modern Pathology 2015; 28: S88-S94

36. Loughrey MB, Shepherd NA. The pathology of bowel cancer screening. Histopathology 2015; 66:66-77

37. Greene FL. Epithelial misplacement in adenomatous polyps of the colon and rectum. Cancer 1974; 33: 206-217
38. Yantiss RK, Bosenberg MW, Antonioli DA, Odze RD, Utility of MMP-1, p53, E-Cadherin, and Collagen IV. Immunohistochemical stains in the differential diagnosis of adenomas with misplaced epithelium versus adenomas with invasive adenocarcinoma. Am J Surg Pathol 2002;26:206-215

\section{How to Cite This Article:}

Geramizadeh B, Marzban M, Owen D. Malignant Colorectal Polyps; Pathological Consideration (A review). Iranian Journal of Pathology. 2017; 12(1):1-8. 